\title{
Evaluation of starch based cryogels as potential biomaterials for controlled release of antibiotic drugs
}

\author{
L P BAGRI, J BAJPAI and A K BAJPAI* \\ Department of Chemistry, Government Autonomous Science College, Jabalpur 482 001, India
}

MS received 13 March 2010

\begin{abstract}
In the present study starch has been blended with poly(vinyl alcohol) to design macroporous architectures following a repeated freeze-thaw method. These macroporous cryogels were loaded with an antibiotic drug, ciprofloxacin hydrochloride (Cfx), and evaluated for its in vitro delivery in a completely controlled manner thus exploring possibilities to use it as a biomaterial in burn or wound healing applications. The key advantage of the present system is that cryogels formed do not contain any chemical crosslinking agent which is often harmful to organic compounds. These Cfx loaded cryogels were characterized by infrared (FTIR) spectroscopy and scanning electron microscopy (SEM) techniques. The controlled release of Cfx drug from cryogels was investigated under varying experimental conditions such as percent loading of the antibiotic drug, chemical architecture of the cryogels and $\mathrm{pH}$, temperature, and nature of the release media. The prepared cryogels show promise to provide a possible pathway for controlling delivery of antibiotic drug thus minimizing the known side effects and improving efficacy also.
\end{abstract}

Keywords. Cryogels; freeze-thaw; drug delivery; macroporous; ciprofloxacin hydrochloride.

\section{Introduction}

Hydrogels are insoluble, three dimensionally crosslinked polymer network structures composed of hydrophilic homo- or hetero-co-polymers, which have the ability to absorb significant amounts of water (Dimitrov 2003). From a biological viewpoint, this is an essential property to achieve an immunotolerant surface and matrix (i.e. with respect to protein adsorption or cell adhesion). Due to the significant water content, hydrogels also possess a degree of flexibility and texture very similar to natural tissue, which minimize potential irritation to surrounding membranes and tissues. The hydrophilic and hydrophobic balance of a gel carrier can be altered to provide tunable contributions which, in turn, influence the diffusive release of drug contained within the gel matrix (Szepes et al 2008; O'Brien et al 2009). Although hydrogels are made up of single polymers that have been extensively used in drug delivery studies, however, in many cases a single polymer alone cannot meet divergent demands in controlled drug release in terms of both properties and performance (Tong et al 2007).

Hydrogels are versatile materials and have potential applications as biomaterials in artificial muscle, drug delivery, contact lenses, dialysis membranes and tissue engineering. However, poor mechanical properties of these materials restrict their applications as structural

\footnotetext{
*Author for correspondence (akbmrl@ yahoo.co.in)
}

biomaterials as in many cases the latter do need tolerable mechanical strength in addition to biophysical characteristics. Crosslinking by chemical agents (McGann et al 2009) or physical method (e.g. ionizing radiation, repeated freezing-thawing etc.) are common methods to produce a hydrogel matrix. The chemical crosslinking is rapid but may involve toxic chemicals whereas physical methods are quite safer and non-toxic but often slow in time scale. The freeze-thaw method is a simple and environment friendly technique to design highly elastic hydrogels (Hyon et al 1994; Peppas et al 1996).

It would be most desirable for a drug release system to match a patient's physiological need at proper time and/or proper site. This is why there is a great interest in the development of such controlled delivery systems $(\mathrm{Wu}$ et al 2001). Drug delivery technology can be brought to the next level by the fabrication of smart materials into single assembled device that is responsive to the individual patient's therapeutic requirements and able to deliver a certain amount of drug in response to biological state. Such smart therapeutics should possess one or more properties such as proper drug protection, local targeting, precisely controlled release, self-regulated therapeutic action, permeation enhancing, enzyme inhibiting, imaging and reporting. This is clearly a highly challenging task and it is difficult to add all of these functionalities in a single device (Juang et al 1996; Elvira et al 2002).

The controlled delivery of drugs has recently become an intensively developed field of pharmaceutical activity. 
The purpose behind controlling the drug delivery is to achieve more effective therapies, less side effects, prolonged efficacy, enhanced safety and reliability of drug therapy, regulated drug release rate and reduced frequency of drug administration to encourage patient compliance while eliminating the potential for both under and over dosing (Qiu and Park 2001).

Ciprofloxacin hydrochloride (Cfx), also called 1-cyclopropyle-6-fluoro-1-4-oxo-7-C-piprazinyl)-3quinoline carboxylic acid hydrochloride monohydrate, has been used as clinical medicine to kill green pus bacillus, large intestine bacillus, strain coccus, streptococcus and gold grape coccus that can result in some type of infections such as respiration, urethra, alimentary canal, etc (He et al 2004). Ciprofloxacin is a leader among the third generation fluoroquinolones with a broad spectrum of antibacterial activity and good penetration in most tissues (Chen et al 1994; Désévauxa et al 2002). Fluoroquinolones such Cfx are considered to be an appropriate choice for the prevention and treatment in infections in bone and soft tissues (Chouhan and Bajpai 2009).

Poly(vinyl) alcohol (PVA) based hydrogels have been used in a number of biomedical applications because of their inherent non-toxicity, non-carcinogenicity, good biocompatibility and desirable physical properties such as rubbery or elastic nature and high degree of swelling in aqueous solutions. PVA has also gained wide pharmaceutical applications as drug-delivery matrices (Korsmeyer and Peppas 1981; Basmadjian and Sefton 1983; Colombo et al 1985) or in the form of powders added to mixtures of other excipients for tablet formation (Thanoo et al 1993).

Starch is composed of two distinct fractions: amylase is the fraction containing about 4000 glucose units and amylopactin is the branched fraction containing about 100000 glucose units. Starch is naturally hydrolyzed by several amylolytic enzymes like $\alpha$-amylase which is an endo enzyme specific to $\alpha-(1,4)-D-$ glucopyranosidic bonds located within polyglucose chains. The degradation products of starch amylolysis are mainly composed of oligosaccharaides, dextrins and maltose (Lia et al 1998).

An exhaustive work has been done in the field of controlled drug delivery of antibiotic drugs through polymeric matrices. The delivery of this drug by starch-PVA macroporous cryogels offers the possibility of maximizing its efficacy and safety and provides a suitable rate of delivery of the therapeutic dose, at the most appropriate site in the body. The drug loaded PVA-starch cryogels matrix may find application as antibiotic patches in postsurgical periods.

\section{Experimental}

\subsection{Preparation of polymer blends}

The freez-thaw method was adopted for preparation of blend hydrogels of starch and PVA as described elsewhere
(Bajpai and Saini 2005). In a typical experiment, definite amounts of starch and PVA were dissolved into distilled water in a Petri-dish and kept at $-20^{\circ} \mathrm{C}$ for $24 \mathrm{~h}$. The frozen gel was then thawed for $1 \mathrm{~h}$ at room temperature and again kept at $-20^{\circ} \mathrm{C}$ for freezing. Such freezing-thawing cycles were repeated at least three times so that the entire mass was converted into a soft, spongy, white blend. The blend so prepared was purified by equilibrating it in distilled water for $72 \mathrm{~h}$ in order to leach out the unreacted chemicals as soon as possible. The swollen blend was cut into smaller discs and dried at room temperature for a week. The dried blend pieces were stored in airtight polyethylene bags.

\subsection{Characterization of blends}

2.2a FTIR spectral analysis: The infrared spectral analysis was performed on an FTIR spectrophotometer (FTIR-8400S, Shimadzu spectrophotometer) by recording the IR spectra of a dry thin film of the blend.

\subsection{ESEM studies}

The morphological features of the cryogels were investigated by recording their scanning electron micrographs (STEREO SCAN, 430, Leica, SEM, USA).

\subsection{Loading of drug}

An important aspect in the use of blends as drug vehicle was the effect of the drug loading level on the drug release rate. Higher drug loading may be achieved either by using highly concentrated drug solution or repeated soaking of blends in the drug solution and then drying them. Ideally, a successful drug delivery system should have a high drug loading capacity thereby reducing the quantity of matrix material for administration. Drug may be bound to the blends either (i) by polymerization in the presence of drug in most cases in the form of solution (incorporation method) or (ii) by absorbing/adsorbing the drug after the formation of blends by incubating them in the drug solution. In the present work, the former method was adopted as in the latter one, however, purification of the loaded device remains a problem (Bajpai and Choubey 2006).

For loading drugs onto the blends, known volume of drug (ciprofloxacin) was taken and diluted with appropriate amount of PBS solution and shaken vigorously for mixing of drug and PBS solution. Drug loaded devices were prepared by allowing $100 \mathrm{mg}$ of blends to swell in freshly prepared drug solution $(10 \mathrm{ml})$ until equilibrium swelling was reached. The percent of drug loaded onto blends was calculated by (1) 


$$
\% \text { Loading }=\frac{W_{\mathrm{d}}-W_{0}}{W_{0}} \times 100,
$$

where $W_{\mathrm{d}}$ and $W_{0}$ are the dry weights of drug loaded and unloaded gels, respectively.

\subsection{In vitro release experiments}

In vitro release experiments were carried out by placing the ciprofloxacin loaded blends $(0 \cdot 1 \mathrm{~g})$ in a test tube containing a definite volume $(10 \mathrm{ml})$ of phosphate buffer saline (PBS) as the release medium $(\mathrm{pH}=7.4)(1.2 \mathrm{mM}$ $\mathrm{KH}_{2} \mathrm{PO}_{4}, \quad 1.15 \mathrm{mM} \quad \mathrm{Na}_{2} \mathrm{HPO}_{4}, 2.7 \mathrm{mM} \quad \mathrm{KCl}, \quad 1.38 \mathrm{mM}$ $\mathrm{NaCl}$ ). The amount of ciprofloxacin released from the cryogel blends was measured spectrophotometrically at $273 \mathrm{~nm}$ (Shimandzu 1700 Pharma Spec.) and the released amount of drug was determined from calibration plot (Knop 1996).

\subsection{Release kinetics}

The suspension of drug loaded blends was shaken for $1.5 \mathrm{~h}$ to study kinetics of the drug release process. In order to monitor the progress of the release process, $5 \mathrm{ml}$ aliquots were withdrawn at desired time intervals and instantly replaced by the same volume of fresh release medium (PBS). In the aliquots withdrawn at different time periods, the amount of drug released was estimated spectrophotometrically.

The release of drug from polymeric blend systems is usually considered as a combination of Fickian (diffusion) and non-Fickian movements of drug molecules through polymer chains (Kosmidis et al 2003). In the present study, the kinetic data were analysed with the help of (2), which could be helpful in determining the mechanism of the release process

$$
W_{\mathrm{t}} / W_{\infty}=K t^{n},
$$

where $W_{\mathrm{t}}$ and $W_{\infty}$ are the amounts of the drug released at time, $t$ and at infinity time (equilibrium amount of drug released), respectively and $K$ is a rate constant. The constants, $K$ and $n$, are characteristics of the drug-polymer system. Hassan and Peppas (2000) were the first to give an introduction to the use and limitations of these equations. For evaluating the diffusion constant of loaded drugs, (3) can be used:

$$
\frac{W_{\mathrm{t}}}{W_{\infty}}=4\left(\frac{D_{t}}{\pi L^{2}}\right)^{0 \cdot 5}
$$

where $D$ is the diffusion constant of the drug and $L$ the thickness of dry blends.

\subsection{Blood compatibility}

In order to evaluate the suitability of a polymer matrix to biomedical applications, particularly as artificial implant, the biomaterial must meet certain criteria prior to its actual realization. Thus, in order to judge the blood compatibility nature of the material, certain test procedures were established for determination of in vitro blood compatibility of the polymer material as discussed below.

2.7a Protein adsorption: The foremost event occurring at the interface of the blood-material contact was the adsorption of plasma proteins (bovine serum albumin, fibrinogen, etc) which was subsequently followed by the adhesion of leukocytes, macrophages or platelets and ultimately leading to fibrous encapsulation. Thus, the adsorption of proteins could be one of the determinants of biocompatible nature of the material. The adsorption of proteins was studied as below.

A known volume of protein solution of definite concentration was mildly shaken with the polymer blend for a definite time period and the remaining concentration of protein was monitored in the solution spectrophotometrically. The amount of the adsorbed protein was calculated according to (4)

$$
\text { Adsorbed amount }\left(\mathrm{mg} \mathrm{m}^{-2}\right)=\frac{\left(C_{0}-C_{\mathrm{a}}\right) V}{A} \text {, }
$$

where $C_{0}$ and $C_{\mathrm{a}}$ being the concentrations of protein solution (mg per $\mathrm{ml}$ ) before and after adsorption, respectively, $V$ the volume of the protein solution and $A$ the surface area of the adsorbent.

2.7b Clot formation test: The antithrombogenic potential of the blends surface was judged by the blood clot formation test as described elsewhere (Bajpai and Kankane 2007). In brief, the starch blends were equilibrated with saline water $(0.9 \% \mathrm{w} / \mathrm{v} \mathrm{NaCl})$ for $72 \mathrm{~h}$ in a constant temperature bath and to these swollen blends, $0.5 \mathrm{ml}$ of acid citrate dextrose (ACD) blood was added followed by the addition of $0.03 \mathrm{ml}$ of $\mathrm{CaCl}_{2}$ solution (4 M) to start the thrombus formation. The reaction was stopped by adding $4.0 \mathrm{ml}$ of deionized water and the formed thrombus was separated by soaking in water for $10 \mathrm{~min}$ at room temperature and then fixed in a $36 \%$ formaldehyde solution $(2.0 \mathrm{ml})$ for another $10 \mathrm{~min}$. The fixed clot was placed in water for $10 \mathrm{~min}$ and weighted after drying.

2.7c Haemolysis assay: Haemolysis experiments were performed on the surfaces of the prepared blends as reported elsewhere (Bajpai and Bundela 2008). In a typical experiment, a dry blend film was equilibrated in normal saline water $\left(0.9 \% \mathrm{NaCl}\right.$ solution) for $24 \mathrm{~h}$ at $37^{\circ} \mathrm{C}$ and human ACD blood $(0.25 \mathrm{ml})$ was added onto the blend films. After $20 \mathrm{~min}, 20 \mathrm{ml}$ of saline was added on the surface to stop haemolysis and the sample was incubated for $60 \mathrm{~min}$ at $37^{\circ} \mathrm{C}$. Positive and negative controls were obtained by adding $0.025 \mathrm{ml}$ of human ACD blood and saline solution, respectively to $2 \mathrm{ml}$ of distilled 
water. Incubated samples were centrifuged for $45 \mathrm{~min}$, the supernatant was taken and its absorbance was recorded on a spectrophotometer at $545 \mathrm{~nm}$. The percent haemolysis was calculated according to (5)

$$
\% \text { Haemolysis }=\frac{A \text { test sample }-A(-) \text { control }}{A(+) \text { control }-A(-) \text { control }},
$$

where $A$ is the absorbance.

\section{Results and discussion}

\subsection{Mechanism of cryogels formation}

Mechanism of hydrogels preparation involves physical crosslinking due to crystallite formation. This method addresses toxicity issues because it does not require the presence of a crosslinking agent. Such physically crosslinked materials exhibit higher mechanical strength than PVA blends crosslinked by chemical or irradiative techniques because in the former case the mechanical load can be distributed along the crystallites of the threedimensional structure (Gruber et al 1974). Although formation of an elastic blend by either having an aqueous solution of PVA at room temperature or successively freezing-thawing a moderately concentrated PVA solution is not a new concept, however, a molecular explanation for this phenomenon is not yet known. Three basic models including hydrogen bonding, polymer crystallites formation and liquid-liquid phase separation have been suggested to explain the mechanisms involved in blend formation.

In the present study, since a mixture of PVA and starch solution was taken, the above three models may be considered to operate simultaneously, thus yielding a highly elastic blend. The reason for this assumption is that starch is a multifunctional biopolymer and its presence in PVA solution will enhance the processes of hydrogen bond making, polymer crystallite formation and phase separation, respectively. The porous nature of blends produced by freezing-thawing method may be explained by the fact that whereas the freezing of a PVA-starch mixture results in the formation of ice crystal domains within the polymer matrix, the thawing process results in melting of the ice crystals, thus leaving wide pores in the blend. A repeated performance of the processes widens the pore sizes and thus enhances the porous nature of the blends. The formation of porous network due to freezingthawing hypothetically is shown in figure 1 .

\subsection{FTIR spectra}

FTIR spectra of the unloaded and Cfx loaded blends composed of PVA and starch are depicted in figures 2(a) and (b), respectively. The FTIR spectra clearly shows a broad band around $3583 \mathrm{~cm}^{-1}$ which is typical of bonded $\mathrm{O}-\mathrm{H}$ stretching of alcoholic hydroxyls of starch and PVA (figure 2(a)). The spectra shows prominent peaks at $2924 \mathrm{~cm}^{-1}$ due to $=\mathrm{CH}$ stretching of methylene group, $1795 \mathrm{~cm}^{-1}$ due to overtone or combination bands, $1483 \mathrm{~cm}^{-1}$ due to $s p^{3}-\mathrm{CH}$ bending, and $1151 \mathrm{~cm}^{-1}$ due to $\mathrm{C}-\mathrm{O}$ stretching of alcohol. The spectra (figure 2(b)) also mark the presence of drug ( $\mathrm{Cfx}$ ) as evident from the observed bands at $1163 \mathrm{~cm}^{-1}(\mathrm{C}-\mathrm{N}$ stretching) and $1003 \mathrm{~cm}^{-1}$ (C-F stretching). Similarly $\mathrm{C}-\mathrm{H}$ out of plane bending vibration of aromatic ring also indicates the presence of $\mathrm{Cfx}$ in the cryogels.

\subsection{Scanning electron microscopy}

The morphological features of the proposed cryogel have been studied by SEM analysis. In order to demonstrate how the freeze-thaw method develops the formation of pores over the cryogels surfaces, a blend of PVA-starch was also prepared by simple solution cast method and the SEM images of both the types of blends were compared.

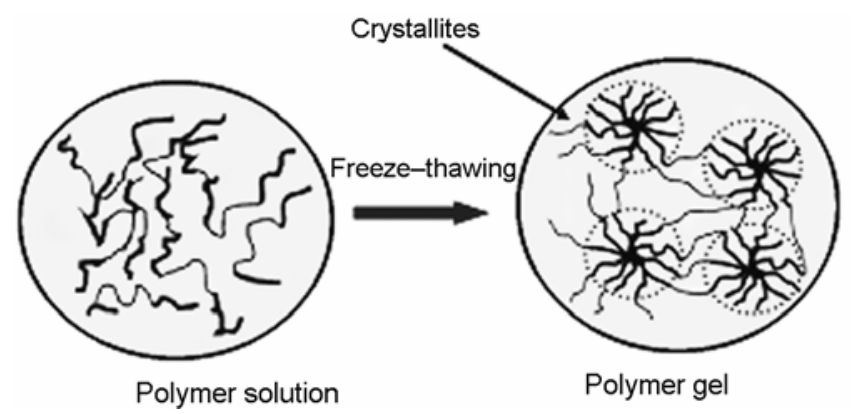

Figure 1. A schematic presentation of mechanism of cryogel formation.

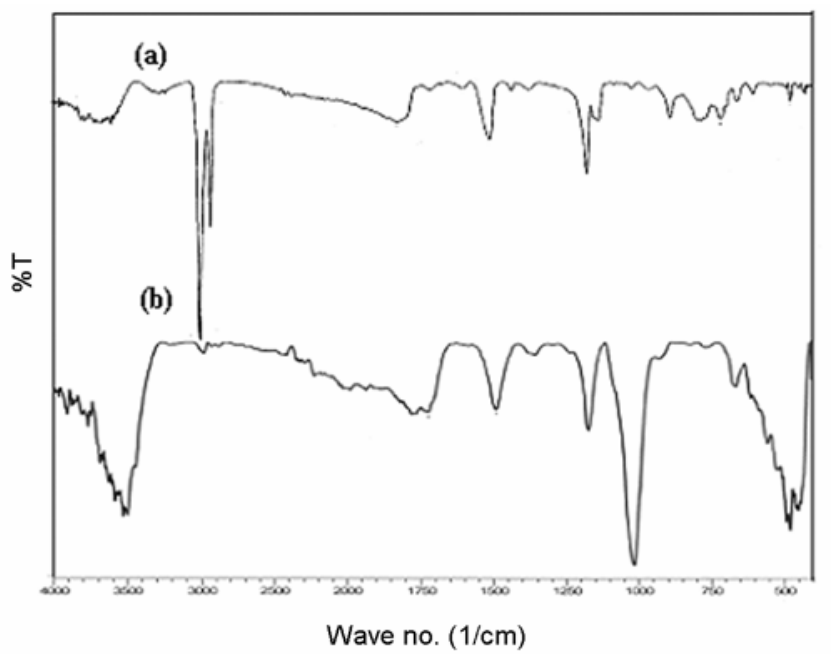

Figure 2. FTIR spectra of (a) Cfx unloaded blend of PVA and starch and (b) Cfx loaded blend of PVA and starch. 

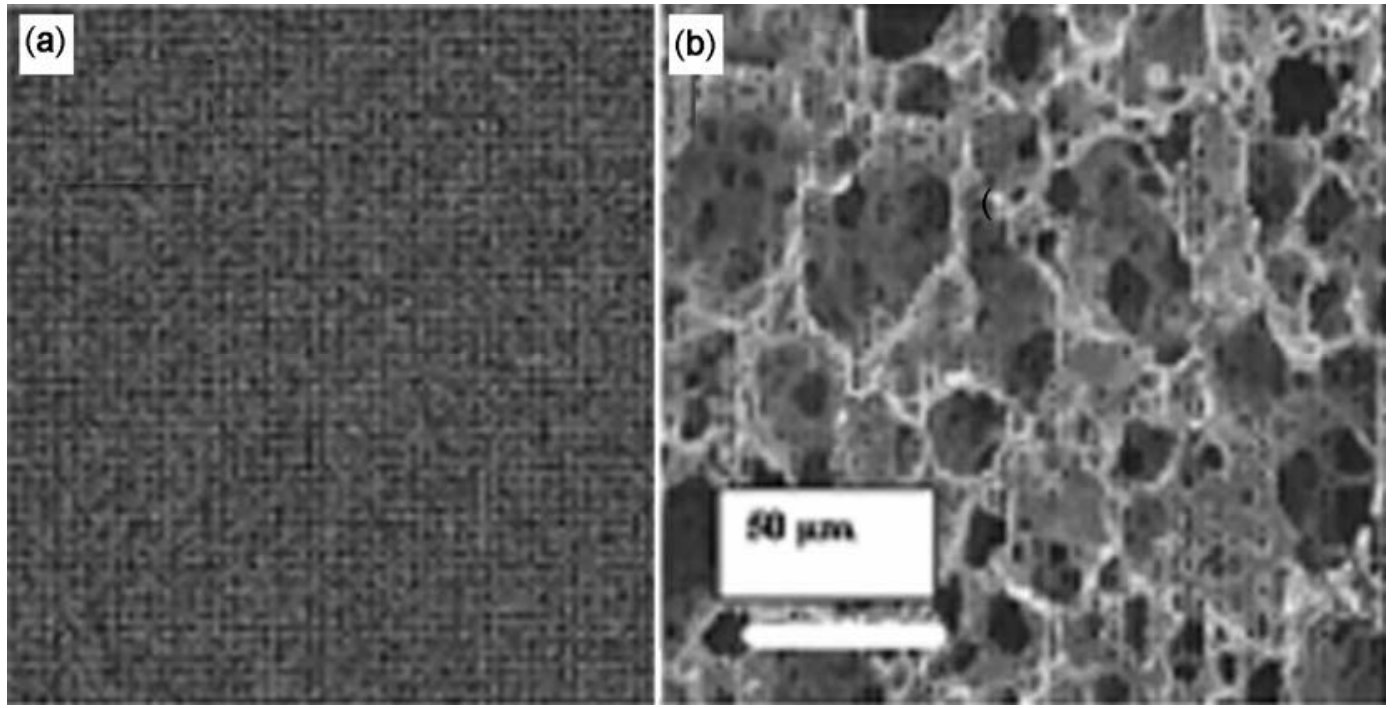

Figure 3. SEM images of blend of PVA and starch synthesized (a) simple solution casting method and (b) freezing-thawing method.

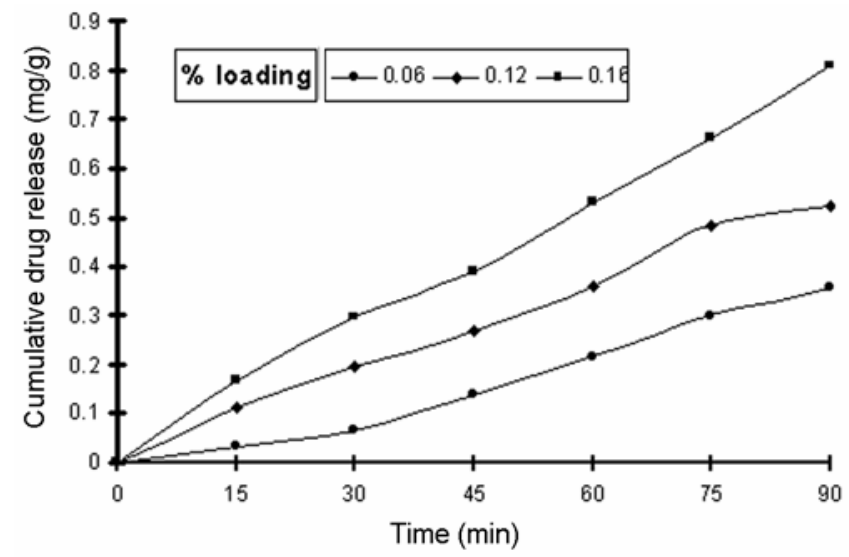

Figure 4. Effect of \% loading on cumulative release of Cfx drug from blend composed of PVA and starch (40/60\% w/w) $\left(\mathrm{pH}=7 \cdot 4\right.$, temp. $\left.=37^{\circ} \mathrm{C}, \mathrm{FTC}=3\right)$.

The SEM images of simple and cryogels blends of PVA and starch are shown in figures 3(a) and (b), respectively. Figure 3(a) clearly shows that both the PVA and starch form a homogeneous blend having no porosity, unevenness, cracks or voids on the surfaces. On the other hand, figure 3(b) presents a porous morphology with pore sizes varying in the range $25-50 \mu \mathrm{m}$. In this way, a clear distinction between the two images provides an experimental evidence for the proposed formation of crystallites during the freezing-thawing method.

\subsection{Effect of \% loading on Cfx release}

In the present work, blends of definite composition were loaded with different amounts of $\mathrm{Cfx}$ by allowing the blends to swell in the drug solution of concentration varying in the range $0 \cdot 5-2 \cdot 0 \mathrm{mg} / \mathrm{ml}$. The loaded blends were allowed to release the entrapped drug into a definite volume of release medium. The release results are shown in figure 4 , which reveal that initially on increasing drug concentration up to $2.0 \mathrm{mg} / \mathrm{ml}$ a larger release of the drug is obtained. The observed results may be attributed to the fact that in the blends with higher loading (up to $2.0 \mathrm{mg} / \mathrm{ml}$ ) of drug concentration, the solvent front (PBS) advances faster into the polymer matrix and, therefore, results in a larger release of the drug. Similar type of results has also been reported by other workers (Roy et al 2009).

\subsection{Effect of wt.\% of composition on drug (ciprofloxacin) releases}

As PVA and starch are the main constituents of the cryogel, their varying weight fraction in the hydrogels exerts a significant influence on the water sorption capacity, as well as other allied properties. Thus, the effect of PVA/ starch on the drug release has been investigated by varying the wt. fraction of PVA in the range 20-71.4 (\% w/w) or that of starch in the range $29-80(\% \mathrm{w} / \mathrm{w})$, respectively. The results are summarized in figure 5, which clearly indicate that the cumulative drug release increase when the weight fraction of the PVA/starch increases from $20 / 80$ to $42 / 58$ while a decrease in the amount of released drug is noticed beyond $42 / 58 \%$ weight fraction of PVA. The observed results may be explained as below.

When the weight percent of PVA increases from 20-42, the tendency of crystallites formation in the blend also increased which results in generation of wide pores in the cryogel blend. These wide pores frequently allow water 
molecules to enter the gel and consequently the Cfx drug molecules dissolve and release out from the blend. However, beyond a certain weight fraction (40/58) of PVA/ starch the blend becomes highly crystalline in nature and, therefore, shows fewer tendencies to absorb water. Thus, due to reduction in swelling the extent of cumulative drug release also decreases.

Another possible reason for the observed decrease may be that increasing wt. fraction of PVA may also produce hydrogen bonding between the polymer chains and may result in highly crosslinked blend network which shows less tendency of water sorption and as a result the amount of released drug decreases.

\subsection{Effect of pH on drug (ciprofloxacin) releases}

For controlled drug delivery system, $\mathrm{pH}$ plays an important role. The utilization of $\mathrm{pH}$ change within the GI tract

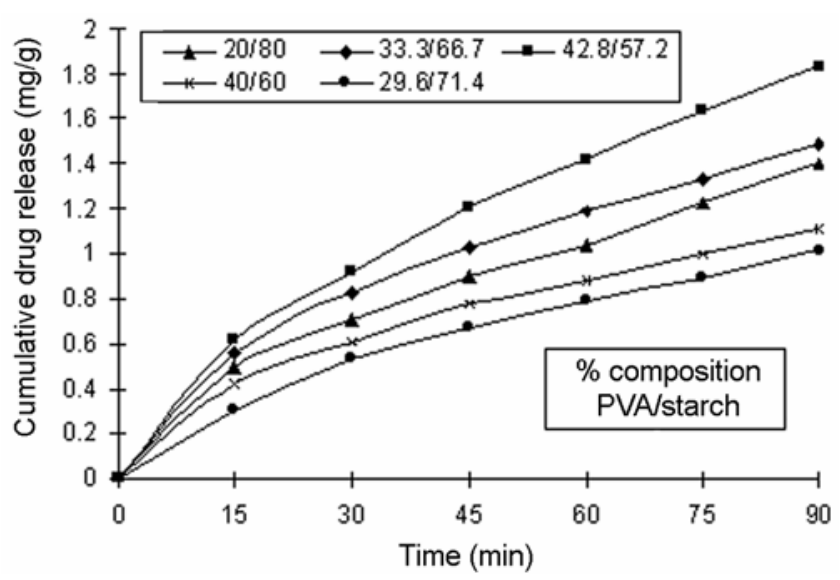

Figure 5. Effect of wt.\% of composition on cumulative release of Cfx drug from blend composed of PVA and starch $\left(\mathrm{pH}=7 \cdot 4\right.$, temp. $=37^{\circ} \mathrm{C}, \mathrm{FTC}=3, \%$ loading $\left.=0 \cdot 16 \%\right)$.

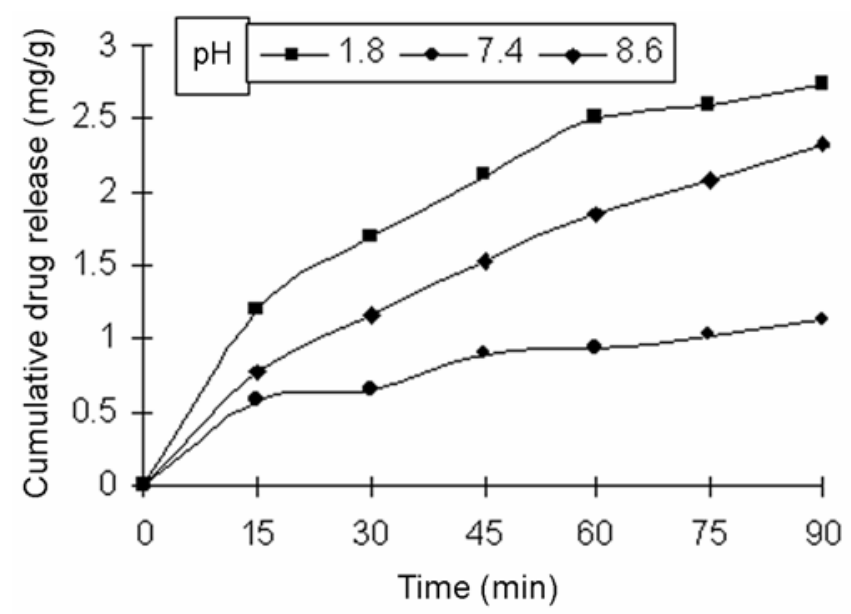

Figure 6. Effect of $\mathrm{pH}$ on cumulative release of $\mathrm{Cfx}$ drug from blend composed of PVA and starch $(40 / 60 \% \mathrm{w} / \mathrm{w})$ (temp. $=37^{\circ} \mathrm{C}, \mathrm{FTC}=3, \%$ loading $=0 \cdot 16 \%$ ). and exploitation of bacterial enzyme localized within the colon is one of current interest in controlled drug delivery. In the present study, the release dynamics of the drug has been observed under varying $\mathrm{pH}$ as found in the GI tract. The release results are depicted in figure 6 which indicates that with increasing $\mathrm{pH}$ of the medium the amount of released Cfx decreases in the range 1.8-7.4 while beyond $7 \cdot 4$, an increase is observed. It means that the release of $\mathrm{Cfx}$ is more at 1.8 (acidic) and 8.6 (basic) medium than $7.4 \mathrm{pH}$. The results can be explained by the fact that the $\mathrm{Cfx}$ (amphoteric in nature) undergoes partial hydrolysis, and consequently produce cationic charged centres $\left(\mathrm{NH}^{2+}\right)$ and these cationic charged centres increase the hydrophilicity of matrix as well as repulsion between cationic charged centres. As a result chains are easily relaxed and thus produced wide free volumes in the network.

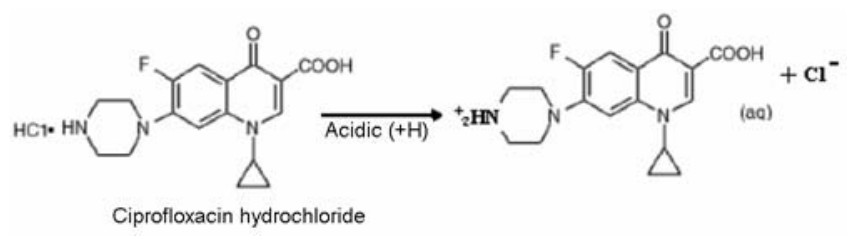

Another reason may be that the sustained release is caused by osmotic pressure difference $\pi_{\text {in }}-\pi_{\text {out }}$ at the inside and outside of the cryogel (Vilches et al 2002). The osmotic pressure $(\pi)$ results from a net difference in concentration of mobile ions between the interior of the nanogel at external medium. Increasing ionic concentration difference obviously results in an enhanced swelling of the polymer which in turn will bring about an increase in the amount of released drug. The osmotic pressure is given as

$$
\pi_{\text {ion }}=R T \sum\left(C_{\mathrm{ig}}-C_{\mathrm{is}}\right),
$$

where $C_{\text {ig }}$ and $C_{\text {is }}$ represent the mobile ion concentration in the cryogel and solution, respectively. When the $\mathrm{pH}$ is $1 \cdot 8$, ionic concentration within the cryogel becomes quite huge. At this $\mathrm{pH}$ due to the $\mathrm{NH}^{2+}$ ion of $\mathrm{Cfx}$, there occurs an increase in differences in ionic concentration between the external and internal medium which results in an increase in the degree of water sorption and this obviously enhances the extent of Cfx release.

Similar types of results have also been obtained in alkali medium. The results can be explained by the fact that the $\mathrm{COOH}$ groups undergo a partial hydrolysis to produce anionic groups, $\mathrm{COO}^{-}$. Now these anions produce repulsive forces and cause macromolecular chains to undergo a fast relaxation which facilitates the penetration of water into the loaded cryogel and subsequently the amount of the released $\mathrm{Cfx}$ increases.

\subsection{Effect of temperature on drug (ciprofloxacin) release}

Temperature sensitivity is one of the most important characteristics in drug delivery technology. It has a direct 
influence on the release behaviour of a hydrogel as it affects both the segmental mobility of the hydrogel chains as well as the diffusion of penetrant molecules. In the present study, the effect of temperature on the drug release from the starch/PVA cryogel has been investigated by varying the temperature of the swelling medium in the range of $5-40^{\circ} \mathrm{C}$. The results are depicted in figure 7, which show that with increasing temperature the released amount also increases.

The observed increase in the released amount of ciprofloxacin can be explained by the fact that with increasing temperature, the network chains undergo faster relaxation due to increased kinetic energy and thus facilitates water sorption process.

\subsection{Effect of number of FTC on drug (ciprofloxacin) releases}

The effect of number of repeated freeze-thaw cycles on the Cfx drug release from cryogels has been studied and

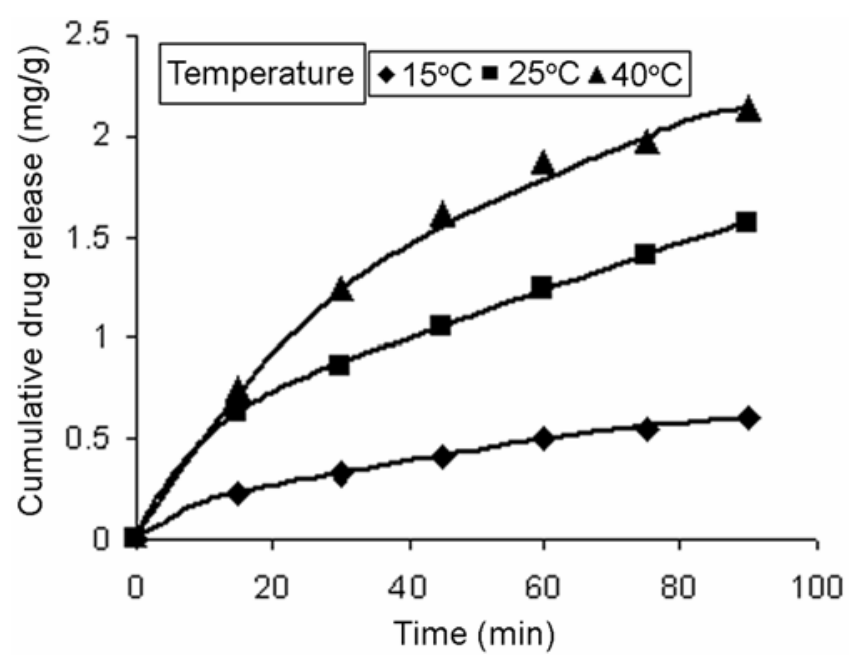

Figure 7. Effect of temperature on cumulative release of $\mathrm{Cfx}$ drug from blend composed of PVA and starch $(40 / 60 \% \mathrm{w} / \mathrm{w})$ $(\mathrm{pH}=7 \cdot 45, \mathrm{FTC}=3$, \%loading $=0 \cdot 16 \%)$.

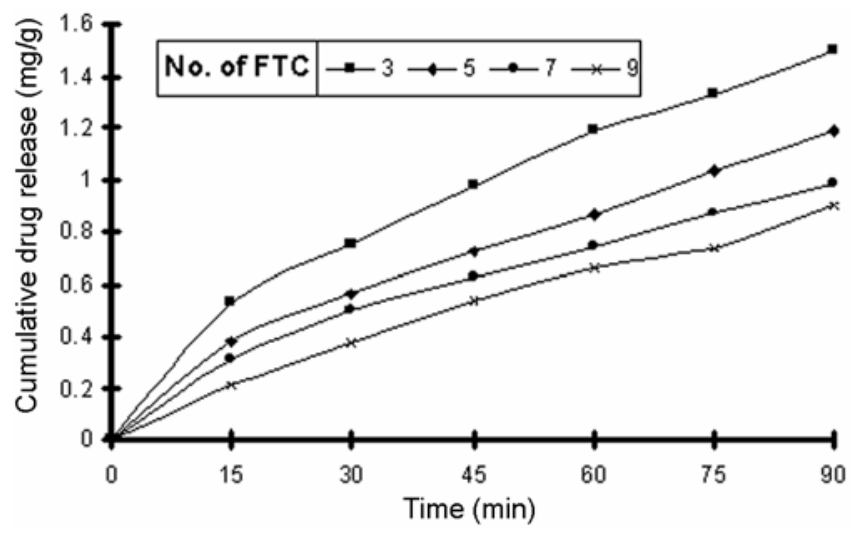

Figure 8. Effect of number of freeze-thaw cycles on cumulative release of Cfx drug from blend composed of PVA and starch $(40 / 60 \% \mathrm{w} / \mathrm{w})\left(\mathrm{pH}=7 \cdot 4\right.$, temp. $=37^{\circ} \mathrm{C}, \%$ loading $\left.=0 \cdot 16 \%\right)$. shown in figure 8 . The results clearly reveal that the extent of drug release decreases with increasing number of FTC. The observed results may be attributed to the fact that in the case of $3 \mathrm{rd}$ cycle, the cryogels does not have large crystalline regions and therefore, the chains of PVA and starch keep on relaxing with increasing releasing time. However, with increasing number of FTC, the gel acquires increasing crystallinity which restricts the mobility of PVA and starch chains and consequently results in a suppressed drug release (Bagri et al 2009).

Another reason for the observed decrease in the amount of drug release with increasing number of FTC is that the pore sizes of the cryogel decreases with increasing number of FTC. Thus, due to shrinkage of the pore sizes of the cryogel the number of incoming water molecules and outgoing drug molecules decreases which results in lower amount of drug release. The reduction in pore sizes with increasing number of FTC is evident from SEM images as shown in figure 9.

\subsection{Effect of physiological fluids on drug (ciprofloxacin) release}

The influence of solutes on the release kinetics of ciprofloxacin was examined by performing release experiments in the presence of solutes such as urea $(5 \% \mathrm{w} / \mathrm{v})$ and D-glucose $(5 \% \mathrm{w} / \mathrm{v})$ and in physiological fluids such as saline water $(0.9 \% \mathrm{NaCl})$ and synthetic urine. The results are depicted in figure 10 which show that the presence of solutes suppresses the drug release. The reason for the lower release of ciprofloxacin in these fluids may be that the presence of salt ions in the release medium lowers the osmotic pressure of the gel and consequently decreases the extent of swelling of loaded blends (Dredán et al 1998).

\subsection{Evaluation of biocompatibility}

The selection of a material to be employed as a biomaterial for a specific end use must meet several criteria such as physicochemical properties, function desired, nature of the physiological environment, adverse effects in the case of failure, expected durability and consideration relating to cost and case of production. Whatever the type of materials, the biocompatibility is the foremost requirement for all biomaterials. In the present study, the assessment of biocompatibility has been made on the basis of three in vitro tests viz. BSA (bovine serum albumin) adsorption test, blood clot formation and haemolysis assay as discussed below.

3.10a BSA adsorption: In the present work, in vitro biocompatibility of prepared blends has been judged by monitoring the amount of protein (BSA) adsorbed by the blend. The results are shown in table 1 , which indicates 

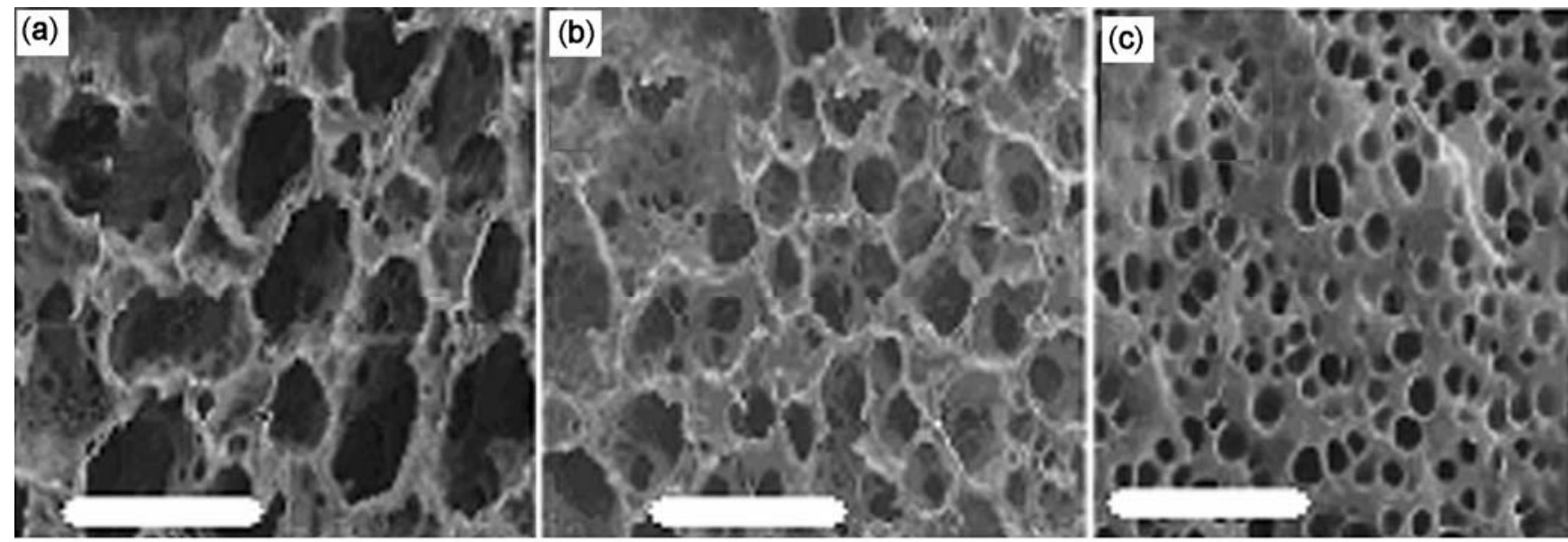

$($ bar $=80 \mu \mathrm{m})$

Figure 9. SEM images depicting reduction in pore sizes of cryogel with increasing number of FTC: (a) 3 , (b) 5 and (c) 7 .

Table 1. Data showing biocompatibility parameters with varying compositions of cryogels.

\begin{tabular}{lcccc}
\hline $\begin{array}{l}\text { PVA/starch \% } \\
(\text { w/w })\end{array}$ & $\begin{array}{c}\text { Freeze-thaw } \\
\text { cycles }\end{array}$ & \% Haemolysis & $\begin{array}{c}\text { BSA adsorbed } \\
\left(\mathrm{mg} \mathrm{g}^{-1}\right)\end{array}$ & $\begin{array}{c}\text { Blood clot } \\
(\mathrm{mg})\end{array}$ \\
\hline $20 / 80$ & 3 & $18 \cdot 19$ & 0.0092 & 0.0008 \\
$33 \cdot 3 / 66 \cdot 7$ & 3 & $16 \cdot 24$ & 0.0075 & 0.0006 \\
$42 \cdot 8 / 57 \cdot 2$ & 3 & $13 \cdot 28$ & 0.0022 & 0.0003 \\
\hline
\end{tabular}

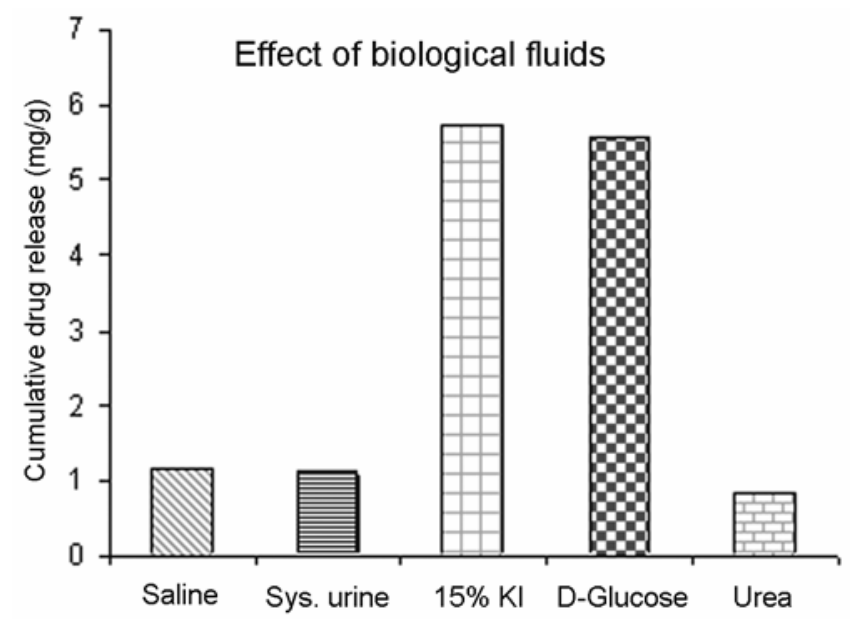

Figure 10. Effect of biological fluids on cumulative release of Cfx drug from blend composed of PVA and starch (40/60 $\% \mathrm{w} / \mathrm{w})\left(\mathrm{pH}=7 \cdot 4\right.$, temp. $=37^{\circ} \mathrm{C}, \mathrm{FTC}=3, \%$ loading $\left.=0 \cdot 16 \%\right)$.

that the amount of adsorbed BSA decreases with increaseing wt. fraction of PVA in the range 20-44 (\% w/w). The observed findings may be explained on the basis of the fact that PVA is non-ionic, biocompatible and hydrophilic polymer and it does not provoke either any damage of blood cells or any change in the surface of plasma proteins which are the main factors for the biocompatibility.

The data summarized in the table also indicates that as the wt. fraction of starch increases in the range 56-80 $(\% \mathrm{w} / \mathrm{w})$, the amount of adsorbed BSA increases which suggests increasing thrombogenicity. The increase observed with increasing starch content may be explained by the fact that with increasing starch content, the wt. fraction of PVA decreases which results in a less biocompatible nature of the matrix. Moreover, increasing starch content may bring about heterogeneity in the blend matrix which may activate adsorption of the protein.

3.10b Blood clot formation: The blood clot experiments were performed on different compositions of the hydrogels and the amount of blood clot formed on the surfaces was recorded. The results are summarized in table 1, which indicate that a much less amount of blood clot is formed. This obviously implies an antithrombogenic nature of the hydrogel surfaces.

3.10c Haemolysis test: The prepared blends were also tested for hemolytic activity and the results obtained are shown in table 1 . The results obtained clearly indicate that with increasing PVA content, the extent of haemolysis constantly decreases. The observed results are attributed to the reason that with increasing wt. fractions of PVA in the blends, the surface composition favourably changes which improves the blood compatible quality of the material.

\subsection{Analysis of kinetic sorption data}

It is a well established fact that the phenomenon of controlled drug release is basically ruled by the water sorption 
Table 2. Data showing variation of diffusional (release) exponent ' $n$ ' and diffusion constant $(D)$ with varying compositions of cryogels.

\begin{tabular}{lccccc}
\hline $\begin{array}{l}\text { PVA/starch \% } \\
(\mathrm{w} / \mathrm{w})\end{array}$ & $\begin{array}{c}\text { Freeze-thaw } \\
\text { cycles }\end{array}$ & Temp. $\left({ }^{\circ} \mathrm{C}\right)$ & $\begin{array}{c}D \times 10^{-6} \\
\left(\mathrm{~cm}^{2} \mathrm{~s}^{-1}\right)\end{array}$ & $n$ & $\begin{array}{c}\text { Release } \\
\text { mechanism }\end{array}$ \\
\hline $20 / 80$ & 3 & 37 & $2 \cdot 41$ & $0 \cdot 52$ & Anomalous \\
$29 \cdot 6 / 71.4$ & 3 & 37 & $2 \cdot 52$ & $0 \cdot 58$ & Anomalous \\
$33 \cdot 3 / 66 \cdot 7$ & 3 & 37 & $2 \cdot 65$ & $0 \cdot 61$ & Anomalous \\
$40 / 60$ & 3 & 37 & $3 \cdot 10$ & $0 \cdot 70$ & Anomalous \\
$42 \cdot 8 / 57 \cdot 2$ & 3 & 37 & $3 \cdot 42$ & $0 \cdot 72$ & Anomalous \\
\hline
\end{tabular}

process that a loaded hydrogel undergoes when immersed into a suitable solvent. The swelling of the hydrogel could be due to the hydration of the polymer which results in a rapid decrease in its glass transition temperature to the temperature of the dissolution medium. Macroscopically, there is a relaxation response of the polymer chains due to stress induced by the presence of the dissolution solvent. This results in an increase in the radius of gyration and end-to-end distance of the polymer chains, causing a significant increase in the molecular volume of the hydrated polymer (Ranga Rao and Devi 1988).

It is clear from table 2 that for cryogel compositions prepared at varying concentration of PVA and starch, the value of $n$ fluctuates between 0.5 and 1 , thus, suggesting an anomalous type of release that is characterized by almost equal rates of solvent diffusion and chain relaxation.

\section{Conclusions}

Repeated freeze-thaw method of preparation of starch and PVA cryogels results in a spongy macroporous polymeric matrix which shows promise to function as swelling controlled drug release system. The structural characterization of prepared blends by FTIR spectral analysis confirms the presence of functional groups of starch, PVA and loaded drug (Cfx) in the blends. The morphology of the blends as studied by SEM analysis suggests the porous nature with pore size ranging from $10 \mu \mathrm{m}$. The addition of model drug, $\mathrm{Cfx}$, to polymeric blends proved to be successful with a $28 \%$ drug entrapment. It is found that release profiles of Cfx are greatly influenced by varying the experimental parameters such as percent loading of Cfx, composition of the cryogel, $\mathrm{pH}$ and temperature of the medium.

With an increase in percentage loading of drug onto the hydrogels, the release of Cfx constantly increases. The released amount of drug constantly decreases with increasing number of freeze-thaw cycles in the cryogels.

An optimum drug release is obtained near $\mathrm{pH} 1.8$ and 8.0 while lower release is noticed in physiological $\mathrm{pH}$ (7.4) range. A rise in temperature up to $40^{\circ} \mathrm{C}$ brings about an increase in the released $\mathrm{Cfx}$ while a decrease is noticed beyond $25^{\circ} \mathrm{C}$. The physiological fluids suppress the extent of drug release. The loaded drug shows retention of its chemical and bioactivity when released into PBS and other $\mathrm{pH}$ media as release mediums. With increasing concentrations of PVA and starch, the biocompatibility of cryogel increases.

\section{References}

Bagri L P, Bajpai J and Bajpai A K 2009 J. Macromol. Sci. Pure \& Appl. Chem. 461060

Bajpai A K and Saini R 2005 Polym. Int. 541233

Bajpai A K and Choubey J 2006 J. Appl. Polym. Sci. 101 2320

Bajpai A K and Kankane S 2007 J. Appl. Polym. Sci. 31559

Bajpai A K and Bundela H 2008 Xpress Polym. Letts 2201

Basmadjian D M and Sefton V 1983 J. Biomed. Mater. Res. 17 509

Chen D H, Leu J C and Huang T C 1994 J. Chem. Technol. Biotechnol. 61351

Chouhan R and Bajpai A K 2009 J. Mater. Sci. Mater. Med. 20 1103

Colombo P, Caramella C, Conte U, Gazzaniga A and La Manna A 1985 J. Controll. Rel. 1283

Désévauxa C, Dubreuilc P and Lenaerts V 2002 J. Controll. Rel. 8283

Dimitrov M 2003 Acta Pharm. 5325

Dredán J, Zelko R, Antal I, Bihari E and Rácz I 1998 Int. J. Pharmaceut. 160

Elvira C, Mano J F, San Roman J and Reis R 2002 Biomaterials 231955

Gruber E, Soehendra B and Schurz J 1974 J. Polym. Sci. Symp. 1444105

Hassan C M and Peppas N A 2000 Adv. Polym. Sci. 15337

He H, Cao X and Lee L 2004 J. Controll. Rel. 95391

Hyon S H, Cha W I, Ikada Y, Kita M, Ogura Y and Honda Y 1994 J. Biomater. Sci. Polym. Ed. 5397

Juang H, Bonner W S, Ogawa Y J, Vacanti P and Weir G C 1996 Transplantation 611557

Knop K 1996 Eur. J. Pharmaceut. Sci. 4293

Korsmeyer R W and Peppas N A 1981 J. Membr. Sci. 9211

Kosmidis K, Argyrakis P and Macheras P 2003 Int. J. Pharm. 254183

Lia J K, Wang N and Wu X S 1998 J. Controll. Rel. 56117

McGann M J, Higginbotham C L, Geever L M and Nugent M J D 2009 Int. J. Pharmaceut. 372154

O'Brien S, Wang Y J, Vervaet C and Remon R J 2009 Carbohyd. Polym. 76557 
Peppas N A, Ratner B D, Haffman A S, Schoen F J and Lemons J E 1996 Biomaterials science: an introduction to materials in medicine (Toronto: Academic) p. 62

Qiu Y and Park K 2001 Adv. Drug Deliv. Rev. 53321

Ranga Rao K V and Devi K 1988 Int. J. Pharm. 481

Roy A, Bajpai J and Bajpai A K 2009 Carbohyd. Polym. 101016

Szepes A, Makai Z, Blümer C, Mäder K, Peter Kasa Jr and Révész P S 2008 Carbohyd. Polym. 72571
Thanoo B C, Sunny M C and Jayakrishnan A 1993 J. Pharm. Pharmacol. 4516

Tong X, Zheng J, Lu Y C, Zhang Z F and Cheng H 2007 Mater. Letts 611704

Vilches A P, Jimenez-Kairuz A, Alovero F, Olivera M E, Allemandi D A and Manzo R H 2002 Int. J. Pharm. 24617

Wu L U, Zhang Y T, Cai C S and Hu S H 2001 New Chem. Mater. 29 